\title{
Covid-19 complicated with chronic necrotizing pulmonary aspergillosis and aspergilloma progressing to fibrosing aspergillosis:
}

a case report

\author{
Zamelina Angela Razafindrasoa ${ }^{1}$, Kiady Ravahatra ${ }^{2}$, Harison Michel Tiaray ${ }^{1}$, Anjara \\ Mihaja Nandimbiniaina ${ }^{1}$, Princy Parfait Andriamahenina ${ }^{1}$, Sonia Marcelle \\ Razafimpihanina ${ }^{1}$, Diamondra ombanjanahary Andriarimanga ${ }^{1}$, Jocelyn Robert \\ Rakotomizao $^{1}$, Joëlson Lovaniaina Rakotoson ${ }^{1}$, and Rondro Nirina Raharimanana ${ }^{2}$ \\ ${ }^{1}$ University of Madagascar \\ ${ }^{2}$ University of Antananarivo
}

March 1, 2022

\begin{abstract}
Cases of aspergillosis superinfection have been reported as acute complications of Covid-19. Here we report a case of combined chronic necrotizing pulmonary aspergillosis, aspergilloma and fibrosing aspergillosis in a 31-year-old woman with a history of Covid-19. This unusual association deserves special attention in a secondary immunocompromised patient after Covid-19.
\end{abstract}

\section{Introduction}

The coronavirus disease 2019 (Covid-19) remains a global concern since its discovery in December 2019. Its course is sometimes unpredictable and can lead to recovery with or without acute complications, long-term sequelae, or death. Acute respiratory distress syndrome, superinfections and disseminated intravascular coagulopathy are the main acute complications in patients with moderate to severe disease [1]. Cases of acute invasive aspergillosis, possibly fatal, have been reported as a complication of Covid-19 in patients with severe form of the disease [2]. To our knowledge, rare cases of subacute invasive aspergillosis or chronic necrotizing aspergillosis have been reported as a complication of Covid-19 pneumonia [3]. Here, we report an unusual combination of chronic necrotizing pulmonary aspergillosis, aspergilloma and fibrosing aspergillosis in a young woman with a history of Covid-19.

\section{Case presentation}

The patient was a 31-year-old housewife. She had a severe form of Covid-19, diagnosed clinically and biologically (positivity of the SARS-CoV-2 RT-PCR test on a nasopharyngeal swab), evolving favourably under a well-conducted treatment including Azithromycin for 5 days, Ceftriaxone, Dexamethasone and Enoxaparine for 14 days. She had no other specific history, including no smoking, no alcoholism, no diabetes and no history of pulmonary tuberculosis. Her history dates back to 3 months after her hospitalization by progressively worsening shortness of breath (dyspnea), left chest pain of a heavy feeling, without radiation, evolving in a context of altered general condition such as asthenia and weight loss. She received various treatments such as antibiotics, tonics and bronchodilators, without any improvement. On the other hand, she developed a productive wet cough with non-fetid yellowish sputum. Then, she was re-hospitalized for respiratory discomfort, chest pain, cough and altered general condition. The clinical examination on admission objectified a decrease in oxygen saturation at $90 \%$ in ambient air (96\% with oxygen 4 litres per minute), a respiratory rate at 20 cycles per minute, a heart rate at 75 beats per minute, a blood pressure at $130 / 60 \mathrm{mmHg}$ and 
a temperature at $36.9^{\circ} \mathrm{C}$. She was asthenic and her body mass index was $20 \mathrm{~kg} / \mathrm{m}^{2}$. The physical examination had nothing particular. The electrocardiogram was unremarkable. Biological examinations revealed an inflammatory syndrome with a hyperleukocytosis of $22.9 \times 10^{9} / 1$, predominantly neutrophils (79\%), a red blood cell sedimentation rate of $24 \mathrm{~mm}$ and a C-Reactive Protein of $13.9 \mathrm{mg} / \mathrm{l}$. Other biological tests were normal, with a D-dimer level of $348 \mathrm{ng} / \mathrm{l}$, a glycaemia of $5.37 \mathrm{mmol} / \mathrm{l}$ and a negative HIV serology. The SARS-CoV-2 RT-PCR test performed on suspicion of a long Covid was negative. SARS-CoV-2 serology was positive for Ig G. The GeneXpert MTB/RIF sputum tests for pulmonary tuberculosis were negative. Sputum cyto-bacteriological tests were also negative. The chest CT scan without injection of contrast agent revealed a cavitary lesion in the apical segment of the left upper lobe, within which was an opacity surmounted by an "air crescent"; typical of fungal ball lesion, suggestive of an aspergilloma (Figure 1). After that, the mycological examination revealed mycelial filaments evoking Aspergillus. It should be noted that aspergillosis serology was not available in the country. On the basis of the clinical, biological, and scan evidences, the diagnosis of probable post-Covid-19 aspergillosis was made. The patient was put on an antifungal treatment with Itraconazole $200 \mathrm{mg}$ per day. The clinical course after 14 days of treatment was favourable with disappearance of dyspnea, reduction of cough, chest pain and asthenia. She was discharged from hospital at the third week of treatment. However, she complained of persistent moderate chest pain. The chest CT scan after 2 months of treatment showed disappearance of the aspergilloma (Figure 2a) with a residual cavity surrounded by parenchymal destruction, suggestive of chronic necrotizing aspergillosis and onset of homolateral fibrosis (Figure 2b). Thus, the same antifungal treatment was continued but a surgical opinion was planned if no improvement was noted. Unfortunately, we lost of follow-up the patient.

\section{Discussion}

In total, three forms of pulmonary aspergillosis, that are chronic necrotizing pulmonary aspergillosis, aspergilloma and fibrosing aspergillosis, were found in a patient with no particular history other than SARS-CoV-2 infection.

By definition, aspergillosis is a fungal infection caused by Aspergillus species. The commonly known manifestations are allergic bronchopulmonary aspergillosis, acute invasive pulmonary aspergillosis, aspergilloma, and chronic pulmonary aspergillosis. The latter includes chronic cavitary aspergillosis, chronic necrotizing aspergillosis, and chronic fibrosis [3,4]. The chronic forms affect immunocompetent or moderately immunocompromised patients. Chronic cavitary disease is usually accompanied by the formation of new (multiple) cavities, but without invasion of the surrounding lung parenchyma [4]. On the other hand, chronic necrotizing pulmonary aspergillosis, also called subacute invasive pulmonary aspergillosis, is more invasive and destroys the surrounding tissue [5]. It is presented in the form of a single thin-walled cavity enlarging over several weeks or months, that may contain or not an aspergilloma [5,6]. In the fibrosing form, the disease progresses quietly with major fibrosing destruction around the cavity [3,4]. According to these definitions, our case corresponds to an aspergilloma that developed in a cavity of chronic necrotizing aspergillosis progressing to the fibrosing form.

Based on experiences with influenza-associated invasive pulmonary aspergillosis, cases of aspergillosis superinfection have been associated with the severe or critical form of Covid-19. However, it is not clear whether SARS-CoV-2 infection is in itself the main risk factor for Covid-19-associated pulmonary aspergillosis, or whether other risk factors, such as corticosteroid therapy frequently used in severe forms, increase the risk of disease progression [7]. Indeed, proinflammatory cytokines and chemokines, such as tumor necrosis factor a (TNFa), interleukin-6 (IL-6), interleukin-10 (IL-10), interleukin-1b and monocyte chemoattractant protein-1 were significantly elevated in patients with severe Covid-19, which would predispose to invasive aspergillosis $[2,7]$. In addition, the use of corticosteroids in the treatment of Covid-19 could also increase the risk of immunosuppression. In our opinion, both the severity of Covid-19 and the corticosteroid therapy induced a moderate immunosuppression that put our patient at risk of chronic aspergillosis.

In the literature, the clinical signs of chronic pulmonary aspergillosis are nonspecific, with or without Covid$19[3,4]$. Respiratory and/or general symptoms are often present for one to six months: cough, sputum, chest pain, dyspnea, hemoptysis with or without long-term fever, altered general condition with sometimes severe 
weight loss $[4,6]$. So additional examinations are necessary to diagnose aspergillosis. There are some criteria to make this diagnosis such as clinical signs, radiological abnormalities, evidence of mycelial filaments, exclusion of alternative diagnoses, failure of antibacterial therapy, clinical response to antifungal therapy and exclusion of severe immunosuppression [8]. Our patient met almost all these criteria.

About the treatment, there is no evidence that the treatment of aspergillosis is different in patients with or without Covid-19 [7]. Indeed, treatment of chronic aspergillosis with or without aspergilloma is based on long-term antifungal agents, including in the first instance Itraconazole 200-400 mg/day or Voriconazole 150$200 \mathrm{mg}$ twice daily, orally, for 3 to 6 months or longer. Surgery is reserved for patients who cannot tolerate medical treatment and for patients with active residual disease despite adequate antifungal treatment [6]. However, according to the European Respiratory Society in 2016, chronic necrotizing aspergillosis or subacute invasive aspergillosis should be treated in the same way as acute invasive pulmonary aspergillosis because of its rapidly progressive nature [9]. In this case, treatment is based on intravenous Voriconazole at a dose of $6 \mathrm{mg} / \mathrm{kg}$ every 12 hours on the first day, followed by $3 \mathrm{mg} / \mathrm{kg}$ every 12 hours and then oral therapy for a total duration of at least 3 months. The evolution of chronic aspergillosis under treatment is variable. The disappearance of aspergilloma is correlated with a favourable clinical response to treatment [5].

\section{Conclusion}

Three forms of pulmonary aspergillosis were found in a single patient with a history of SARS-CoV-2 infection. This rare association deserves special attention in an immunocompromised patient caused both by the treatment and the proinflammatory cytokines secreted during Covid-19. In this reported case, the diagnosis was made lately. The clinical symptoms are misleading. So to improve the management of patients, the performance of a chest CT scan remains crucial when faced with persistent respiratory symptoms after SARS-CoV-2 infection. Furthermore, mycological examination should be deepened, especially in resourcelimited countries like ours.

\section{Consent for publication}

After the second chest CT scan, we could obtain a written consent from the patient to allow us to publish a report of her case for educational purposes. She has been de-identified in this manuscript and no personally identifiable details have been included.

\section{Conflict of interest}

The authors declare no conflict of interest.

\section{Authors'contributions}

ZAR, KR, HMT: draft of the clinical case report

AMN, FPPA, SMR, DOA: follow-up of the patient

JRR, JLR, RNR: final correction

All authors read and approved the final manuscript.

\section{References}

[1] Huang C, Wang Y, Li X, Ren L, Zhao J, Hu Y et al. Clinical features of patients infected with 2019 novel coronavirus in Wuhan, China. Lancet . 2020;395(10223):497-506.

[2] Lai CC, Yu WL. COVID-19 associated with pulmonary aspergillosis: A literature review. J Microbiol Immunol Infect . 2021;54(1):46-53.

[3] Patti RK, Dalsania NR, Somal N, Sinha A, Mehta S, Ghitan M et al. Subacute Aspergillosis "Fungal Balls" Complicating COVID-19. J Investig Med High Impact Case Rep . 2020;8:2324709620966475.

[4] Blandin S, David G. L'aspergillose en pratique pour le pneumologue. Rev Pneumol Clin . 2008;64(4):202210. 
[5] Kanj A, Abdallah N, Soubani AO. The spectrum of pulmonary aspergillosis. Respir Med . 2018;141:121131.

[6] El-Baba F, Gao Y, Soubani AO. Pulmonary Aspergillosis : What the Generalist Needs to Know. Am J Med . 2020;133(6):668-674.

[7] Koehler P, Bassetti M, Chakrabarti A, Chen SCA, Colombo AL, Hoenigl M et al. Defining and managing COVID-19-associated pulmonary aspergillosis : the 2020 ECMM/ISHAM consensus criteria for research and clinical guidance. Lancet Infect Dis . 2021;21(6):e149-e162.

[8] Binder RE, Faling LJ, Pugatch RD, Mahasaen C, Snider GL. Binder et al. Chronic necrotizing pulmonary aspergillosis: a discrete clinical entity. Medicine . 1982;61:109-24.

[9] Denning DW, Cadranel J, Beigelman-Aubry C, Ader F, Chakrabarti A, Blot S et al. European Society for Clinical Microbiology and Infectious Diseases and European Respiratory Society. Chronic pulmonary aspergillosis: rationale and clinical guidelines for diagnosis and management. Eur Respir J . 2016;47(1):4568.

\section{Legends}

Figure 1: Parenchymal window chest CT scan, without contrast agent injection, showing a thin-walled cavitary lesion containing a homogeneous opacity with "crescent air" sign, suggestive of aspergilloma (arrow) in the apical segment of the left upper lobe

Figure 2a: Parenchymal scan of the chest 2 months after antifungal treatment, showing a thin-walled, regular empty cavity in the left upper lobe (brown arrow)

Figure 2b: Parenchymal scan of the chest 2 months after antifungal treatment, showing the residual cavity surrounded by more or less heterogeneous opacities (yellow arrows) suggestive of a necrotizing lung lesion and the onset of homolateral fibrosis

\section{Hosted file}

Figure 1.docx available at https://authorea.com/users/445466/articles/558182-covid19-complicated-with-chronic-necrotizing-pulmonary-aspergillosis-and-aspergillomaprogressing-to-fibrosing-aspergillosis-a-case-report

\section{Hosted file}

Figure 2a.docx available at https://authorea.com/users/445466/articles/558182-covid19-complicated-with-chronic-necrotizing-pulmonary-aspergillosis-and-aspergillomaprogressing-to-fibrosing-aspergillosis-a-case-report

\section{Hosted file}

Figure 2b.docx available at https://authorea.com/users/445466/articles/558182-covid19-complicated-with-chronic-necrotizing-pulmonary-aspergillosis-and-aspergillomaprogressing-to-fibrosing-aspergillosis-a-case-report 